\title{
High-Temperature Electroluminescence of InGaN/GaN Light-Emitting Devices with Multiple Quantum Barriers
}

\author{
Ya-Fen Wu \\ Department of Electronic Engineering, Ming Chi University of Technology, Taishan, New Taipei City 243, Taiwan \\ Correspondence should be addressed to Ya-Fen Wu, yfwu@mail.mcut.edu.tw
}

Received 27 August 2012; Accepted 27 September 2012

Academic Editor: Donghui Li

Copyright () 2012 Ya-Fen Wu. This is an open access article distributed under the Creative Commons Attribution License, which permits unrestricted use, distribution, and reproduction in any medium, provided the original work is properly cited.

We investigate the high-temperature characteristics of InGaN/GaN multiple quantum well light-emitting devices with and without multiple quantum barriers (MQBs) in depth. The electroluminescence measurements were carried out over a temperature range from 200 to $380 \mathrm{~K}$ and an injection current level from 1 to $100 \mathrm{~mA}$. Enhanced carrier confinement and stronger carrier localization in the active layer are achieved for the sample with MQBs. Furthermore, it is found that the external quantum efficiency of the sample possessing MQBs is higher than that of the sample with GaN barriers. The MQB structure improves the high-temperature operation of light-emitting devices.

\section{Introduction}

The progress in the epitaxial growth and device processing of GaN-based heterostructures has made this material system a promising light emitter operated in the wavelength regime ranging from infrared to ultraviolet [1-3]. Much interest has been focused on InGaN/GaN multiple quantum wells (MQWs) because they are key structures for the highbrightness blue-green light-emitting diodes (LEDs) and laser diodes (LDs) [3, 4]. The understanding of optical properties of MQW structure is very important for the further improvement in the performance of the devices. In spite of the existence of high-density $\left(\sim 10^{10} / \mathrm{cm}^{2}\right)$ misfit dislocations, the InGaN MQW LEDs shows very bright emission characteristics. Thus, origins of the high quantum efficiency have been receiving much attention. The quantum confinement effects on the InGaN alloy well and efficient carrier capturing by the localized radiative recombination centers in the quantum-dot-like states have been claimed to be important for origins of the high emission efficiency. It has been known that potential fluctuation, originating from segregation effect in $\mathrm{InGaN}$, is one of the important reasons for the exciton localization effect in InGaN/GaN MQWs.

Because of large lattice and thermal expansion coefficient mismatches between the sapphire substrate and the epitaxial layers, the InGaN-based heterostructures display misfit dislocation in nanocrystalline structures, which lead to nonradiative recombination centers. The performance and power output of such devices are limited by the thermally activated loss of electrons overflowing from the active layer to the nonradiative recombination centers, thus reducing the emission efficiency. Moreover, for the lighting applications, the InGaN/GaN MQW devices are confronted by the requirements of high current injection. High current operation in LEDs causes self-heating and raises the junction temperature, further degrading the quantum efficiency. To improve the carrier blocking effect, an AlGaN thin film layer has been used in the multiple quantum wells (MQWs) region of high-power UV/blue/green LEDs to achieve high device efficiency $[5,6]$. An alternative approach is to use multiple quantum barrier (MQB) structures to inhibit hot carrier overflow [7-9]. This technique was based on the use of a superlattice which consisted of alternating thin layers of narrow and wide bandgap semiconductors materials. The MQB structure has been proposed to enhance carrier confinement of semiconductors lasers by virtually increasing the barrier height, using the interference of the reflected electron wave from the boundaries of stacked superlattices [7-9].

In this study, we investigate the high-temperature characteristics of InGaN/GaN MQWs light-emitting devices 
with GaN barriers and with MQB structures. The MQW samples were prepared by metal-organic vapor phase epitaxy system. The electroluminescence (EL) measurements were carried out over a high-temperature range from 200 to $380 \mathrm{~K}$ and an injection current level from 1 to $100 \mathrm{~mA}$. From the measured results of EL spectra, it is found that the sample with MQBs exhibits a less temperature-sensitive characteristic and a stronger localization effect. The external quantum efficiency of the sample with MQBs is significantly higher than that of the sample with GaN barriers at room temperature. The experimental results indicate that the MQB structures could not only improve the carrier recombination, but also profoundly improve the high-temperature operation of light-emitting devices.

\section{Experiment}

Figure 1 shows a schematic diagram of the layered structure investigated in this work. The samples were grown by metalorganic vapor phase epitaxy (MOVPE) on $c$-plane sapphire substrates. Ammonia $\left(\mathrm{NH}_{3}\right)$, trimethylgallium (TMG), trimethylindium (TMI), silane $\left(\mathrm{SiH}_{4}\right)$, and bis(cyclopentadienyl)magnesium ( $\mathrm{Cp}_{2} \mathrm{Mg}$ ) were used either as precursors or as dopants. The layer structure of the sample with $\mathrm{GaN}$ barriers consisted of a 20-nm-thick GaN buffer layer, a 3$\mu \mathrm{m}$-thick Si-doped n-type GaN layer, an undoped GaN layer possessing five periods of $\operatorname{In}_{0.18} \mathrm{Ga}_{0.82} \mathrm{~N} / \mathrm{GaN} \mathrm{MQWs}$, and a 100-nm-thick Mg-doped p-type GaN layer. The doping concentrations of $\mathrm{n}$ - and p-type GaN were nominally about $5 \times$ $10^{18} \mathrm{~cm}^{-3}$ and $1 \times 10^{19} \mathrm{~cm}^{-3}$, respectively. The thicknesses of the InGaN wells and the GaN barriers in the MQW structures were $2 \mathrm{~nm}$ and $11 \mathrm{~nm}$, respectively. For the sample with MQBs, the layer structure was similar, but the MQWs were five periods of $\operatorname{In}_{0.15} \mathrm{Ga}_{0.85} \mathrm{~N} / \mathrm{GaN}$ MQWs, and the barrier layer was replaced by a five-period $\operatorname{In}_{0.005} \mathrm{Ga}_{0.995} \mathrm{~N} / \mathrm{GaN}$ $(1 \mathrm{~nm} / 1 \mathrm{~nm})$ heterolayers. In the MQW and MQB structures, the Indium composition was determined by photoluminescence and X-ray diffraction measurements [10]. The lightemitting diode chips were fabricated over a broad area $\left(350 \times 350 \mu \mathrm{m}^{2}\right)$ using standard photolithography processes. $\mathrm{Ti} / \mathrm{Al} / \mathrm{Ti} / \mathrm{Au}$ and $\mathrm{Ni}-\mathrm{Au}$ were employed for the $\mathrm{n}$ - and $\mathrm{p}$ type electrodes, respectively. For temperature-dependent EL measurements, the samples were mounted in a closed-cycle He cryostat, where the temperature $(T)$ was varied from 200 to $380 \mathrm{~K}$ and using a current source operated from 1 to $100 \mathrm{~mA}$. The luminescence signal was dispersed through a $0.5 \mathrm{~m}$ monochromator and detected using a Si photodiode by standard lock-in amplification technique.

\section{Results and Discussion}

Figure 2 shows the evolution of EL spectra for the samples over a temperature range from 200 to $380 \mathrm{~K}$ at the injection current level $10 \mathrm{~mA}$. We observed InGaN-related emissions with peak energies 2.69 and $2.93 \mathrm{eV}$ at room temperature for samples with GaN barriers and with MQBs, respectively. Referring to the conduction band profile of MQB structure shown in Figure 3, the MQB structure is engineered to align a forbidden energy miniband of the MQB

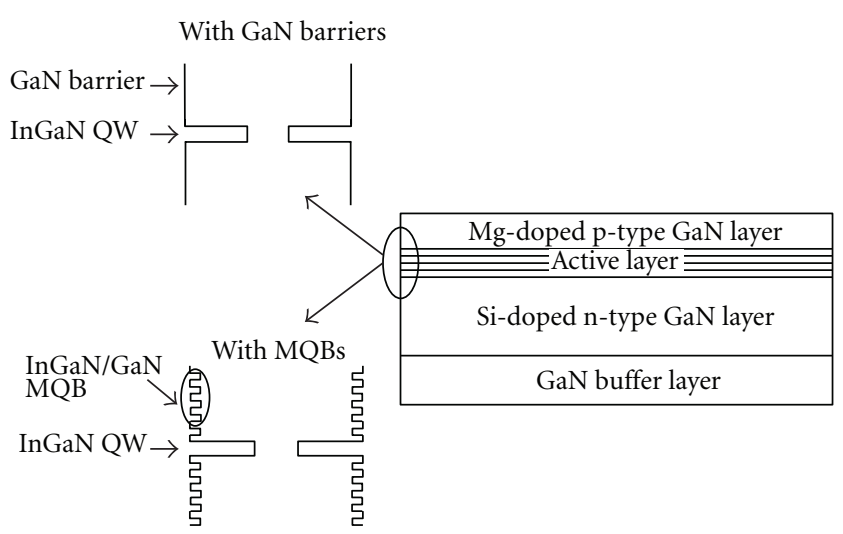

Figure 1: Schematic structure of InGaN/GaN multiple quantum well light-emitting devices (a) with GaN barrier and (b) with multiquantum barriers.

superlattice with the conduction band minimum to enhance the conduction band discontinuity. By using the transfer matrix method to solve the Schrödinger equation, one can obtain the reflectivity probability against incident electron energy. The result illustrates that a band of nonallowed electron states can be created above the classical barrier height $U[9,11]$. This effectively enhances the reflectivity of electrons incident on the barriers and hence, increases the band discontinuity by $\mathrm{d} U$. The higher EL peak energy of this sample with MQBs gives the evidence of the increased conduction band discontinuity. Besides, the higher EL spectra intensity observed for the sample with MQBs in Figure 2(b) could be attributed to the improvement of carrier confinement and inhibited carrier leakage in the active layer. With increasing temperature, both samples exhibit redshift of emission peaks due to the bandgap shrinkage which is resulting from the temperature induced dilation of the lattice and electron-lattice interaction. From the figure, we observe the amounts of redshift from 200 to $380 \mathrm{~K}$ for samples with GaN barriers and with MQBs are $18.5 \mathrm{meV}$ and $15.2 \mathrm{meV}$, respectively, implying a less temperaturesensitive characteristic of the MQB sample. An S-shaped temperature dependence of the EL peak energy (redshiftblueshift-redshift) has been reported to be a fingerprint of the existence of the localization effect based on the bandtail-filling model for thermalized carriers with a Gaussian distribution of energy potential fluctuation and dispersion $[12,13]$. In our measurements the blueshift is not observed since the temperature-dependent EL spectra were measured in the high-temperature range. The blueshift of peak energy induced resulting from the increasing temperature which enables carriers at the localized energy states to populate the higher levels is compensated and overcome by the redshift of the emission peak wavelength due to the band gap narrowing caused by increasing temperature.

To further investigate the luminescence mechanism, the driving current-dependent EL measurements were carried out. Figure 4 describes the EL spectra of the sample with $\mathrm{GaN}$ barriers in the driving current range 1 to $100 \mathrm{~mA}$ at different temperatures. With increasing injection current, 


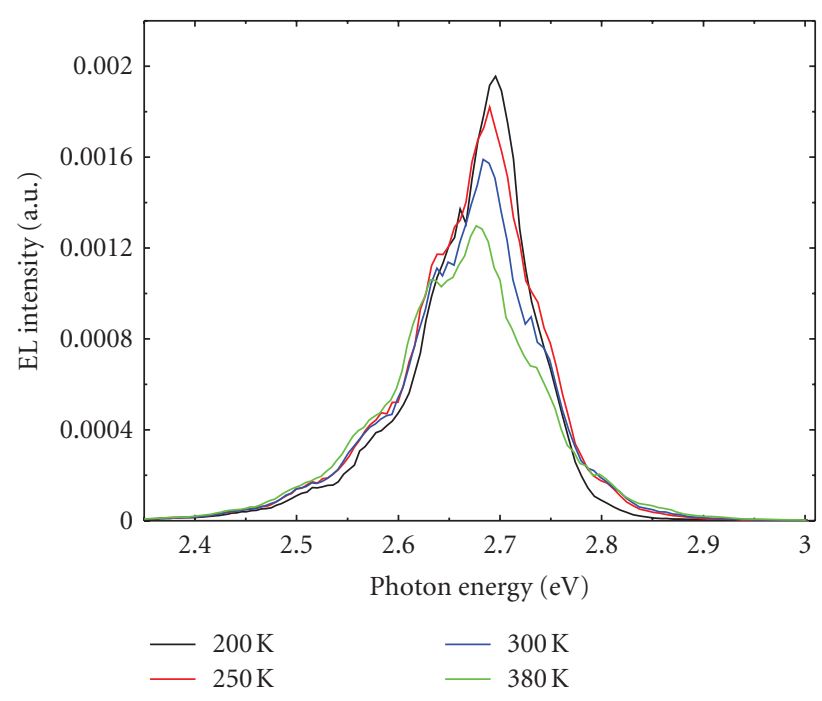

(a)

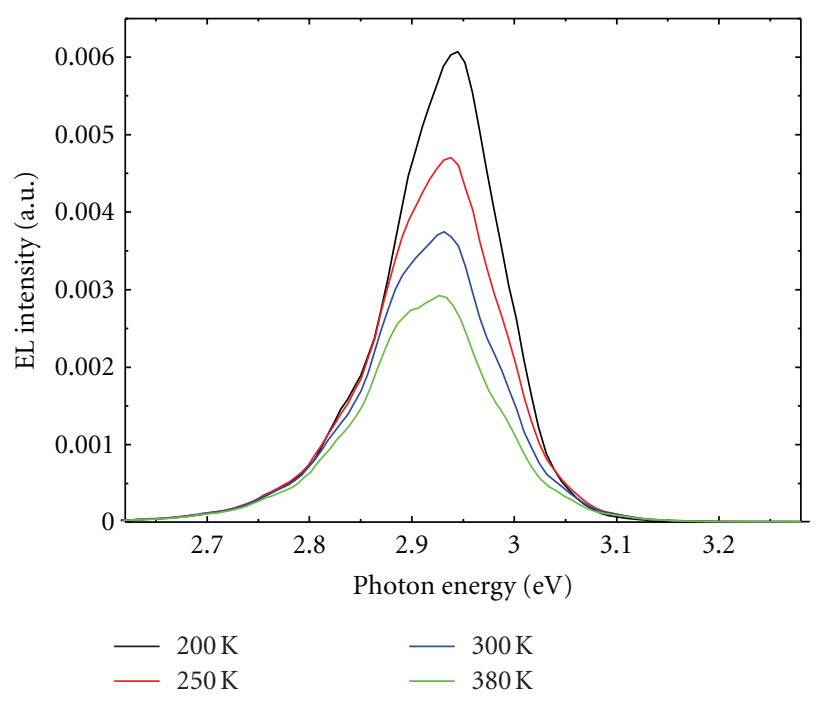

(b)

FIgURE 2: EL spectra of InGaN/GaN MQWs in the temperature range from 200 to $380 \mathrm{~K}$ for (a) sample with GaN barriers and (b) sample with MQBs. The driving current is $10 \mathrm{~mA}$.

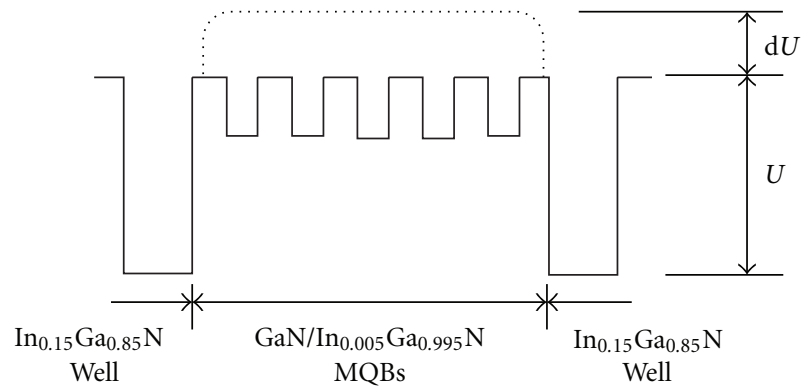

Figure 3: Schematic diagram showing potential energy profile of the MQB structure, where $U$ and $\mathrm{d} U$ are the classical and enhanced barrier height, respectively.

the band-filling effect of the localized energy states caused by indium composition fluctuation in the MQW structures and the screening of piezoelectric field induced quantumconfined Stark effect (QCSE) have been reported for nitridebased structures [14-16]. The QCSE exists in GaN-based MQWs due to the strong piezoelectric fields arising from the strain caused by lattice mismatch between the barrier and well material. Generally, the strain-induced polarization field tilts the potential profile, which results in a redshift of the emission energy. Besides, this field induces a spatial separation of the electron and hole wave functions in the well. The wave function overlap decreases, and the interband recombination rate decreases. When the samples are driven with a current source, the carrier screening of the QCSE flattens the potential across the MQWs [17]. Both bandfilling and QCSE-screening effects should induce an increase in luminescence intensity and a blueshift of emission peak of the EL spectra. The measured results for the sample with GaN barriers at $200 \mathrm{~K}$ are shown in Figure 4(a). As the injection current increases from 1 to $100 \mathrm{~mA}$, the intensities of the EL spectra increase. There is no change in the emission peak energy as the current is lower than $60 \mathrm{~mA}$, and then a redshift appears as the current is further increased to $100 \mathrm{~mA}$. It is reasonable to infer that the blueshift of EL peak energy predicted by raising the driving current is compensated and overcome by the thermally activated bandgap shrinkage, thus a shift to lower energy side for the EL emission peaks is observed. The redshifts of EL peak energy shown in Figures 4(b) and 4(c) are more visible because the sample was operated at higher temperatures, and the heating effect is evident. Furthermore, at injection current $100 \mathrm{~mA}$, the EL spectra intensity becomes saturated, which is ascribed to the thermally-activated nonradiative recombination of the injected carriers.

A different injection current-dependent behavior of EL spectra is observed for the sample with MQBs presented in Figure 5. Owing to the higher indium content of this sample the effect of the indium-rich regions on the EL spectra is remarkable. Referring to the curves shown in Figures 5(a), 5 (b), and 5(c) which measured at 200, 300 and $380 \mathrm{~K}$, respectively, at low injection currents only a change in shape was observed, and no significant shift of peak energy of the spectra was observed. As the injection current increases to $80 \mathrm{~mA}$, in Figure 5(a), the EL intensity saturates and in Figures 5(b) and 5(c) it starts to degrade. The current where the intensity drops is lower than that of the sample with GaN barriers. Thanks to crystal quality decreases with the increased indium content, the degree of the overall potential fluctuations in the InGaN composition and the roughness of the quantum well interface are enhanced. This sample exhibits more nonradiative recombination centers, which in turn, causing a faster intensity quenching rate at high injection current levels. However, even operated at high injection 


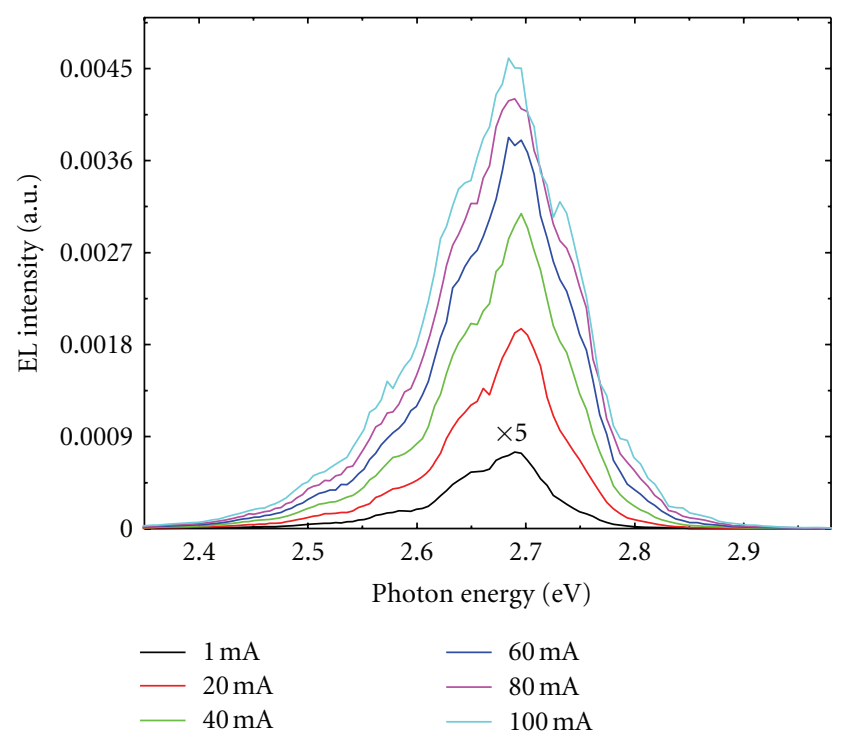

(a) $T=200 \mathrm{~K}$

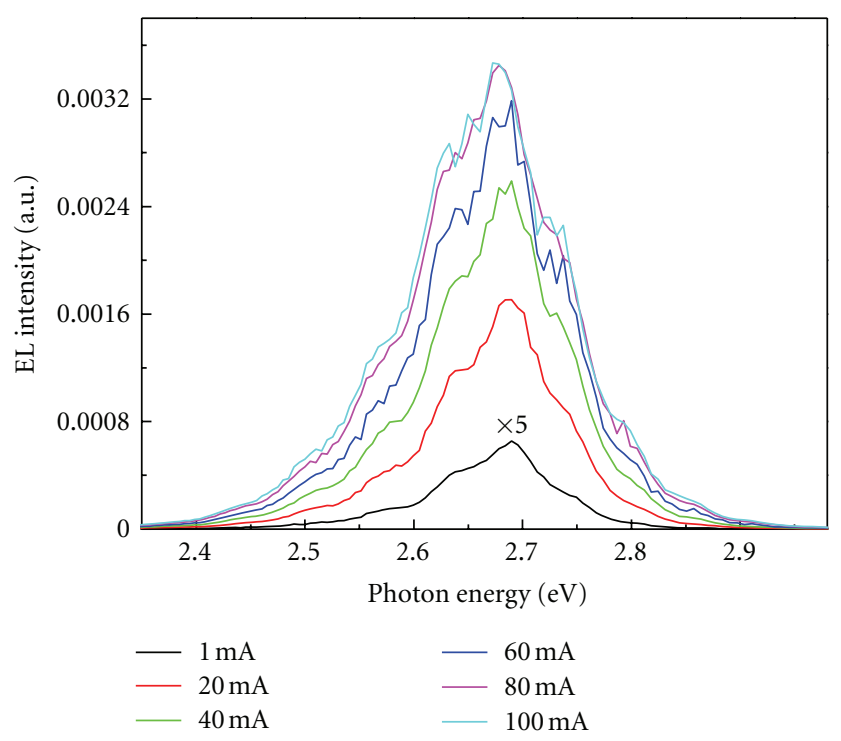

(b) $T=300 \mathrm{~K}$

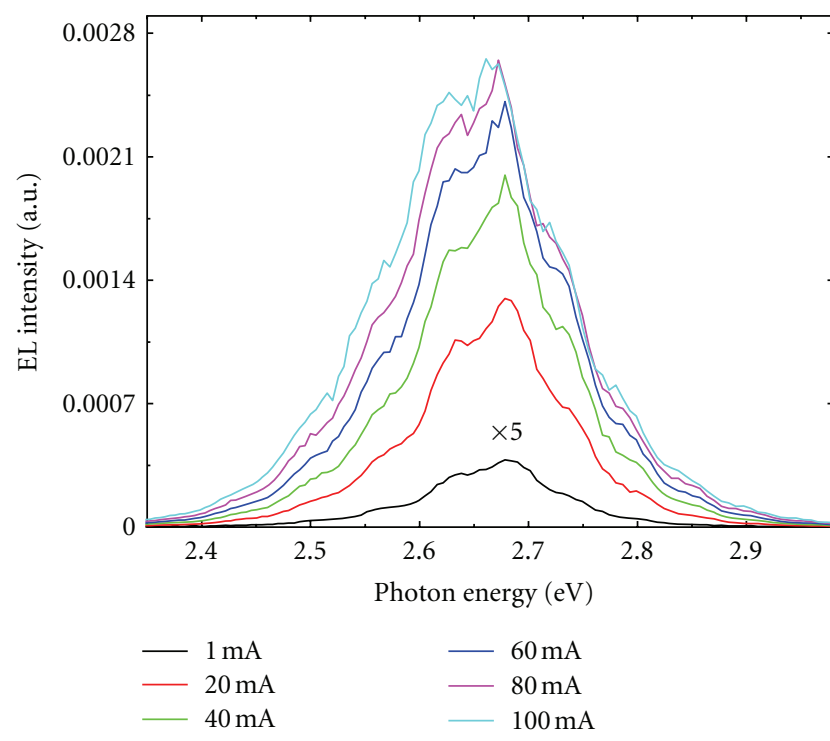

(c) $T=380 \mathrm{~K}$

Figure 4: The EL spectra of sample with GaN barriers in the driving currents range from 1 to $100 \mathrm{~mA}$ at temperature (a) $200 \mathrm{~K}$, (b) $300 \mathrm{~K}$, and (c) $380 \mathrm{~K}$.

current, the EL intensity of MQB sample is higher than that of the sample with GaN barriers at room temperature.

In Figures 5(b) and 5(c), there are anomalous behaviors of driving current dependence of EL spectra. Interestingly, with increasing currents, the dominant emission peak changed from higher energy to lower energy indicates a radiative recombination of excitons localized in the quantumdot-like indium-rich regions. Since the sample is with higher indium content, more localized states may exist and yield a stronger localization effect of it. The higher energy side emission is thought to be the radiative recombination of excitons confined in the regular MQWs, and the lower energy side emission is from the recombination of localized excitons in the MQWs. We decompose the EL signals into two
Gaussian-shaped peaks, and the intensities of the emission peaks from higher energy and lower energy are denoted as $I_{\text {high }}$ and $I_{\text {low }}$, respectively. In the high injection current range the heating effect is apparent, leading to the increasing nonradiative recombination of carriers. Injected carriers that are localized in indium-rich regions prevent them from reaching nonradiative recombination sites. The higher energy emission is quenched quickly, and the lower energy emission becomes dominant in the EL emission spectra. More evidences are shown in Figure 6, where the EL intensity of emissions from higher energy $\left(I_{\text {high }}\right)$ and lower energy $\left(I_{\text {low }}\right)$ are presented separately as a function of driving current at different temperatures. The influence of $I_{\text {low }}$ on the EL intensity becomes conspicuous as the 


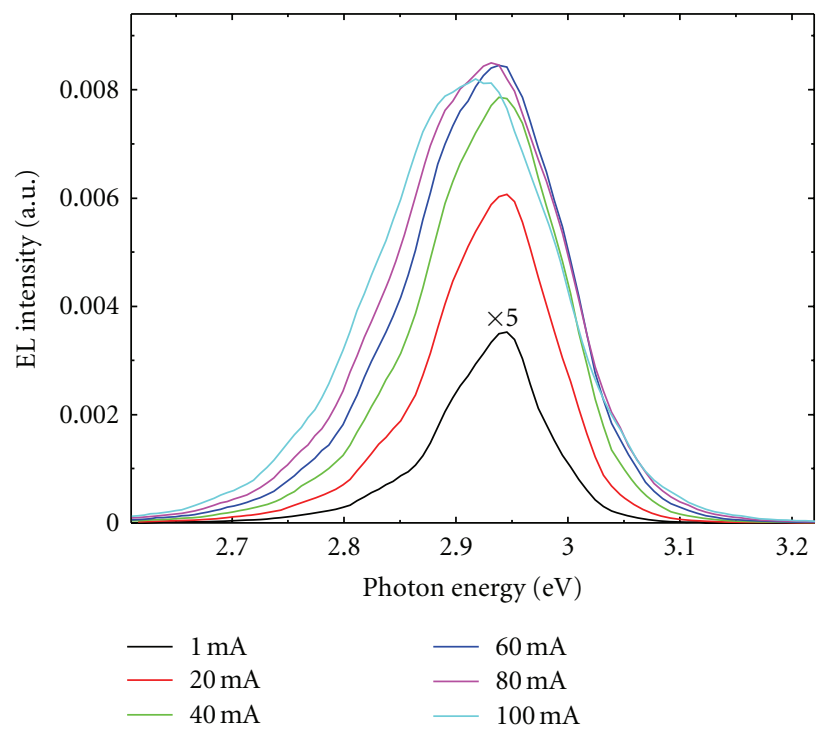

(a) $T=200 \mathrm{~K}$

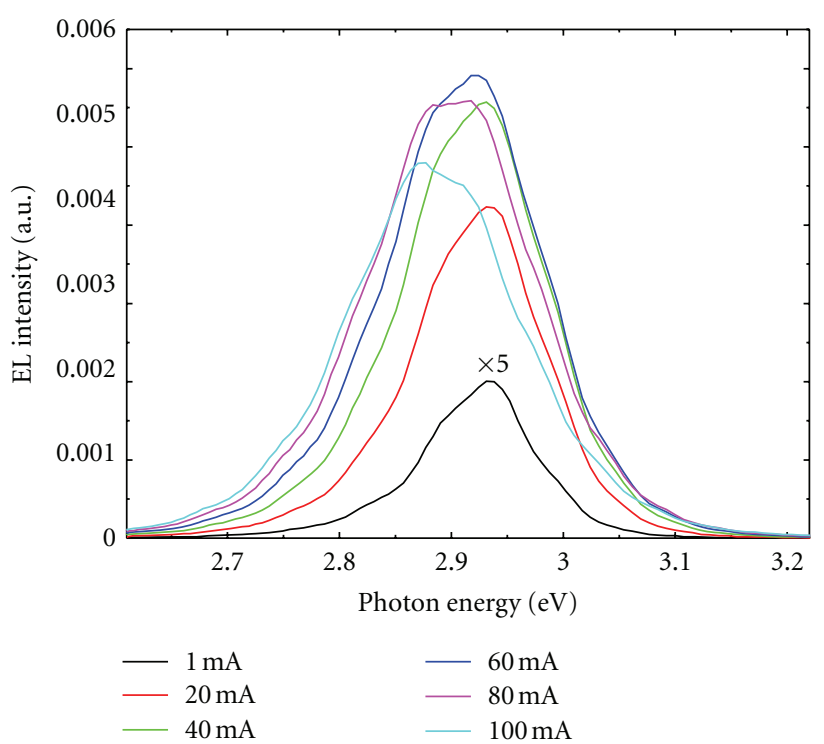

(b) $T=300 \mathrm{~K}$

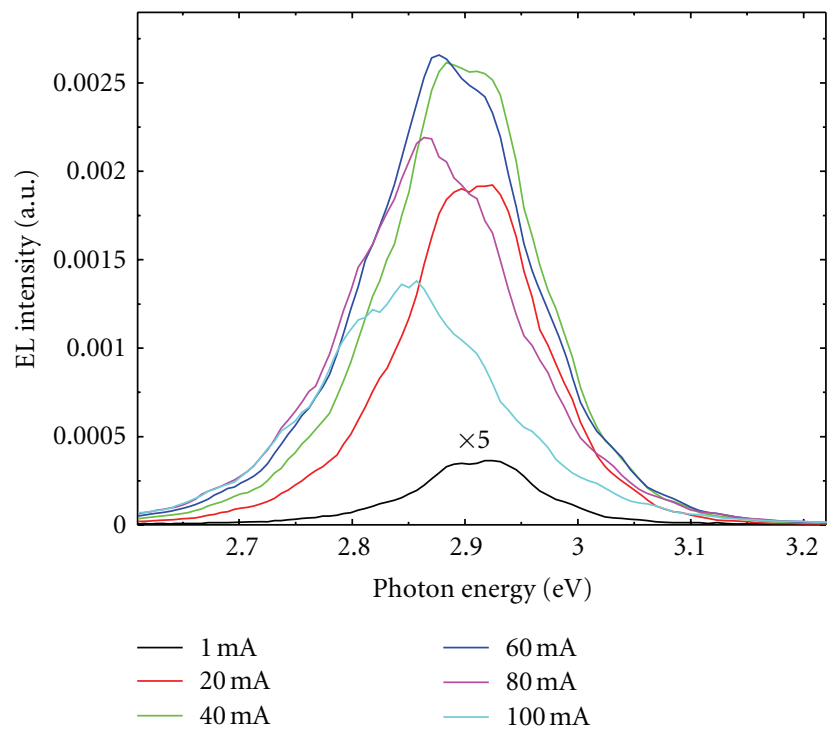

(c) $T=380 \mathrm{~K}$

FIGURE 5: The EL spectra of sample with MQBs in the driving currents range 1 to $100 \mathrm{~mA}$ at temperature (a) $200 \mathrm{~K}$, (b) $300 \mathrm{~K}$, and (c) $380 \mathrm{~K}$.

current goes up, especially in the high-temperature region. It implies that the localization effect in the sample with MQBs is evident. The stronger localization effect, together with the higher conduction band discontinuity, lead to the higher EL intensity for the sample with MQBs.

The effect of MQB structures on the performance of our samples is further investigated by discussing the EL efficiency of the samples. The EL external quantum efficiency of the InGaN/GaN MQW light-emitting diode is also influenced by the increasing temperatures and increasing driving currents. One of the major problems of InGaN/GaN light-emitting devices is the suppression of carrier overflow from the active layer into the p-layer. Because the MQB structure effectively increases the band discontinuity, it is expected to prevent the electrons from overflowing as operated at high temperatures and high driving currents. In order to show detailed variations of the EL efficiency as a function of injection current at different temperatures for the samples, the integrated EL intensity is divided by current (denoted by $\eta_{\text {eff-ex }}$ ), which is proportional to the EL external quantum efficiency. For the injection current $10 \mathrm{~mA}$, the calculated $\eta_{\text {eff-ex }}$ ratio of sample with MQBs to sample with GaN barriers as a function of temperature is shown in Figure 7(a). It is clearly seen that the efficiency of the sample with MQBs is greatly improved. The external quantum efficiency of sample with MQBs exhibits up to 3.5 times higher than that of the sample with GaN barriers, implying that an improved light-emitting device performance can be achieved by the adoption of an appropriate MQB structure. As the injection current is raised from 10 to $100 \mathrm{~mA}$, the $\eta_{\text {eff-ex }}$ ratio gradually 


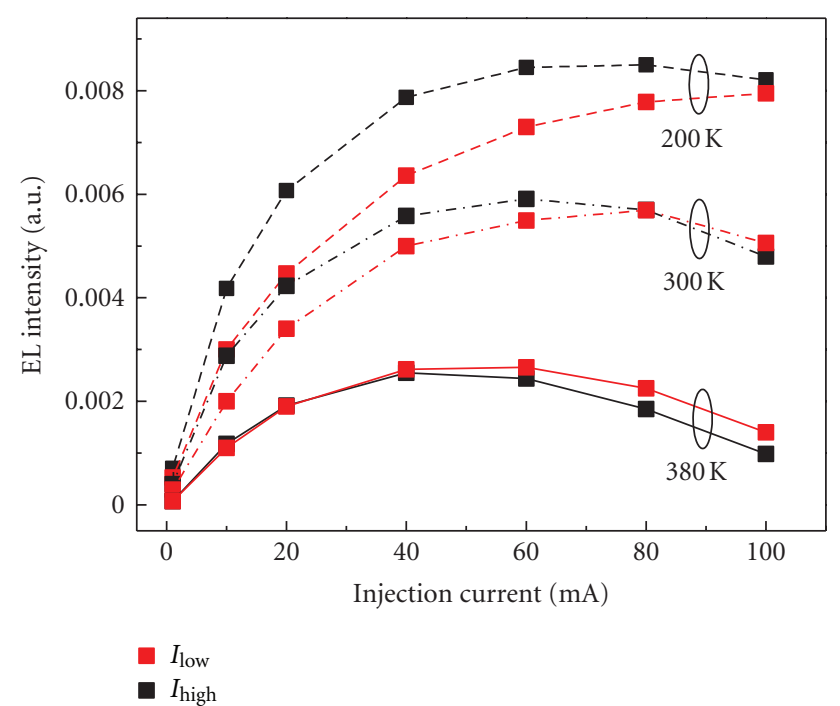

FIGURE 6: Driving current-dependent EL intensity of emissions from higher energy $\left(I_{\text {high }}\right)$ and lower energy $\left(I_{\text {low }}\right)$ at temperature 200,300 , and $380 \mathrm{~K}$, respectively.

dropped, as shown in Figure 7(b). Nevertheless, the external quantum efficiency of sample with MQBs is greater than the sample with $\mathrm{GaN}$ barriers until the injection current is larger than $80 \mathrm{~mA}$, and the temperature is higher than $340 \mathrm{~K}$. Due to the intensified nonradiative recombination of carriers for the MQB sample, the luminescence efficiency exhibits a rapid degradation in the high temperature and high driving current range. As discussed above, the presence of more inhomogeneous composition of indium in this sample paves the way for localized carriers from the MQBs and quantum well to contribute to rapid quenching of efficiency at high temperature. However, as far as the external quantum efficiency at room temperature and high driving current up to $100 \mathrm{~mA}$, the sample with MQBs essentially exhibits the better device characteristics than the sample with GaN barriers.

\section{Conclusion}

In this paper, we investigate the performance of InGaN/GaN MQW LEDs with GaN barriers and with MQBs by the measurements of EL spectra over a broad range of driving current 1 to $100 \mathrm{~mA}$ and temperature range 200 to $380 \mathrm{~K}$. From the detailed analysis of the experimental results, the sample with MQBs exhibits an enhancement of carrier confinement and a stronger localization effect in the active layer coincides with the increase of effective barrier heights and the higher indium content of the sample. Moreover, from the evaluation of injection current-dependent EL external quantum efficiency at room temperature, the sample with MQBs exhibits a greater efficiency as the driving current increases from 10 to $100 \mathrm{~mA}$. It is concluded that the MQB structure improves the performance of InGaN/GaN MQW light-emitting devices.

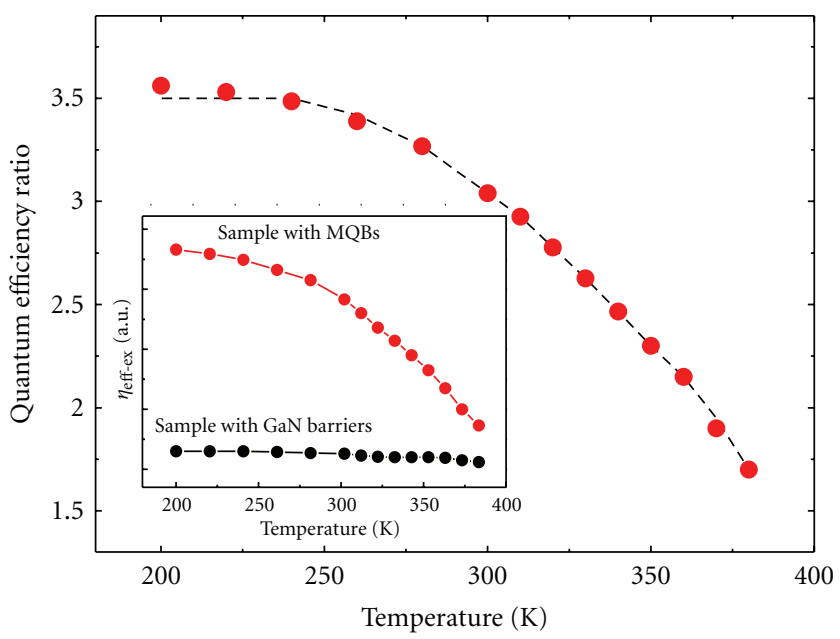

(a)

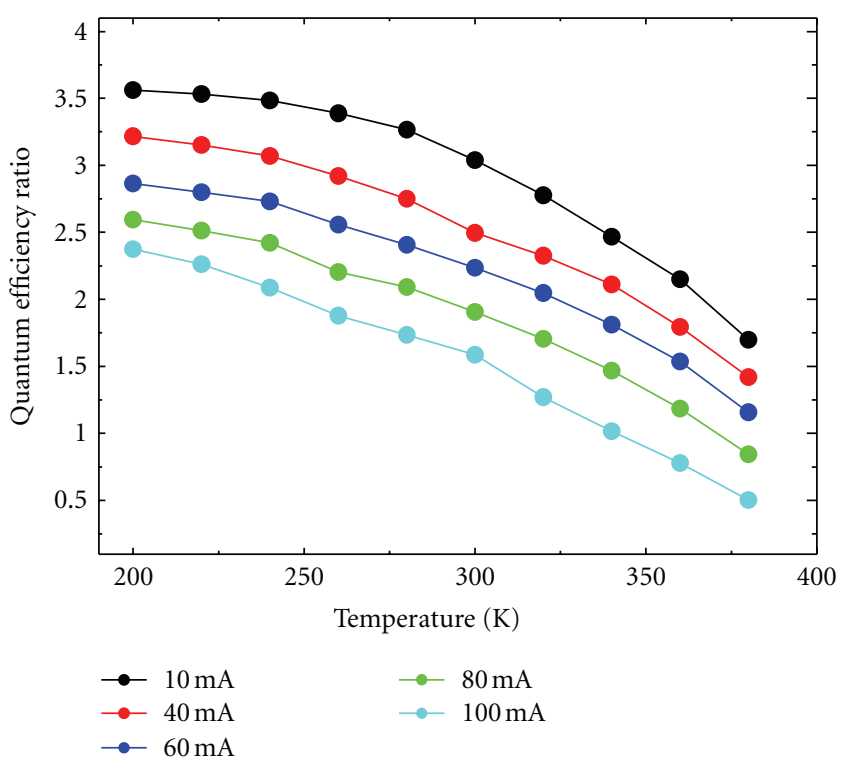

(b)

Figure 7: (a) Temperature-dependent EL quantum efficiency ratio of the sample with MQBs to the sample with GaN barriers. The inset shows the dependence of the EL efficiency on temperature for the samples. The driving current is $10 \mathrm{~mA}$. (b) Temperature-dependent EL quantum efficiency ratio of the samples in the driving current range 10 to $100 \mathrm{~mA}$.

\section{Acknowledgment}

The author would like to thank Mr. Jen-Cheng Wang for his technical assistance.

\section{References}

[1] S. Nakamura, S. Pearton, and G. Fasol, The Blue Laser Diode, Springer, Berlin, Germany, 2000.

[2] E. F. Schubert and J. K. Kim, "Solid-state light sources getting smart," Science, vol. 308, no. 5726, pp. 1274-1278, 2005.

[3] H. Wang, Z. Ji, S. Qu et al., "Influence of excitation power and temperature on photoluminescence in InGaN/GaN multiple 
quantum wells," Optics Express, vol. 20, no. 4, pp. 3932-3940, 2012.

[4] M. Brendel, A. Kruse, H. Jönen et al., "Auger recombination in GaInN/GaN quantum well laser structures," Applied Physics Letters, vol. 99, no. 3, Article ID 031106, 2011.

[5] R. M. Lin, S. F. Yu, S. J. Chang, T. H. Chiang, S. P. Chang, and C. H. Chen, "Inserting a p-InGaN layer before the p-AlGaN electron blocking layer suppresses efficiency droop in InGaNbased light-emitting diodes," Applied Physics Letters, vol. 101, no. 8, Article ID 081120, 4 pages, 2012.

[6] C. H. Jang, J. K. Sheu, C. M. Tsai, S. C. Shei, W. C. Lai, and S. J. Chang, "Effect of thickness of the p-AlGaN electron blocking layer on the improvement of ESD characteristics in GaN-Based LEDs," IEEE Photonics Technology Letters, vol. 20, no. 13, pp. 1142-1144, 2008.

[7] K. S. Teng, M. Brown, A. Kestle et al., "An investigation of multi-quantum barriers for band offset engineering in AlGaInP/GaInP lasers," Applied Surface Science, vol. 190, no. 1-4, pp. 284-287, 2002.

[8] M. P. Ackland, P. R. Dunstan, M. R. Brown, K. S. Teng, S. P. Wilks, and R. Cobley, "Characterisation of the influence of multi-quantum barrier reflectors within GaInP/AlGaInP quantum well lasers using near-field imaging techniques," Applied Surface Science, vol. 255, no. 3, pp. 649-652, 2008.

[9] T. E. Nee, J. C. Wang, H. T. Shen, and Y. F. Wu, "Effect of multiquantum barriers on performance of InGaN/GaN multiplequantum-well light-emitting diodes," Journal of Applied Physics, vol. 102, no. 3, Article ID 033101, 7 pages, 2007.

[10] J. C. Lee, "Investigation of nanostructure properties in InGaN/ GaN multiple quantum wells by X-ray diffraction analysis," Solid State Sciences, vol. 13, no. 5, pp. 1013-1016, 2011.

[11] Y. Y. Lin, R. W. Chuang, S. J. Chang et al., "GaN-based LEDs with a chirped multiquantum barrier structure," IEEE Photonics Technology Letters, vol. 24, no. 18, pp. 1600-1602, 2012.

[12] Y. F. Wu, J. C. Lee, T. E. Nee, and J. C. Wang, "Carrier localization effect on luminescence spectra of IIIV heterostructures," Journal of Luminescence, vol. 131, no. 7, pp. 1267-1271, 2011.

[13] C. Y. Chen, C. Hsieh, C. H. Liao et al., "Effects of overgrown p-layer on the emission characteristics of the InGaN/GaN quantum wells in a high-indium light-emitting diode," Optics Express, vol. 20, no. 10, pp. 11321-11335, 2012.

[14] T. Akasaka, H. Gotoh, T. Saito, and T. Makimoto, "High luminescent efficiency of In GaN multiple quantum wells grown on InGaN underlying layers," Applied Physics Letters, vol. 85, no. 15, pp. 3089-3091, 2004.

[15] N. Khan and J. Li, "Effects of compressive strain on optical properties of $\operatorname{In}_{x} \mathrm{Ga}_{1-x} \mathrm{~N} / \mathrm{GaN}$ quantum wells," Applied Physics Letters, vol. 89, no. 15, Article ID 151916, 3 pages, 2006.

[16] D. Y. Lee, S. H. Han, D. J. Lee et al., "Effect of an electron blocking layer on the piezoelectric field in InGaN/GaN multiple quantum well light-emitting diodes," Applied Physics Letters, vol. 100, no. 4, Article ID 041119, 4 pages, 2012.

[17] S. De, A. Layek, S. Bhattacharya et al., "Quantum-confined stark effect in localized luminescent centers within InGaN/ GaN quantum-well based light emitting diodes," Applied Physics Letters, vol. 101, no. 12, Article ID 121919, 5 pages, 2012. 

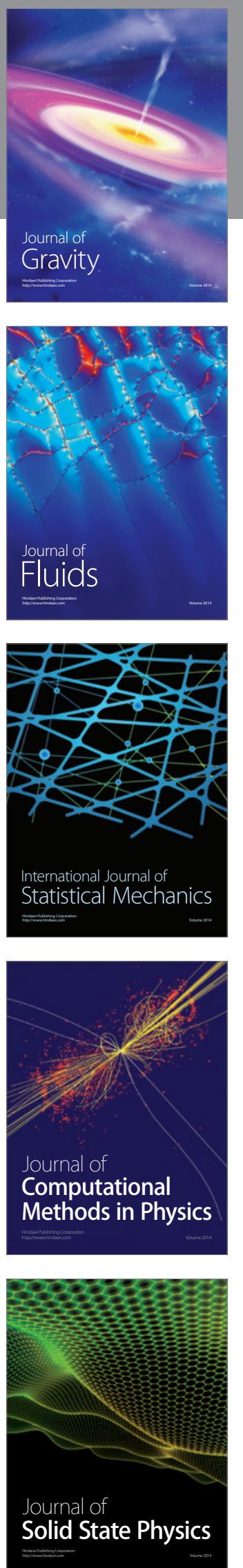

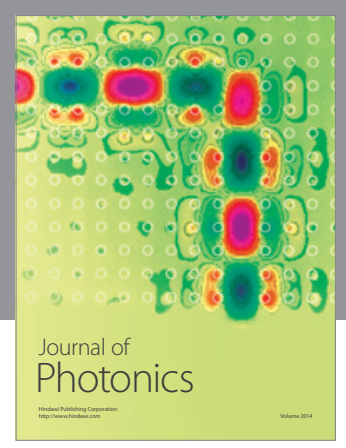

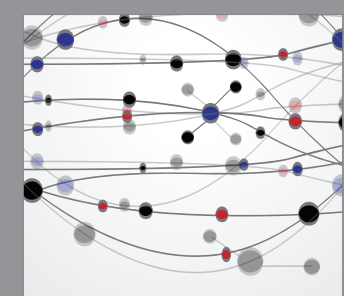

The Scientific World Journal
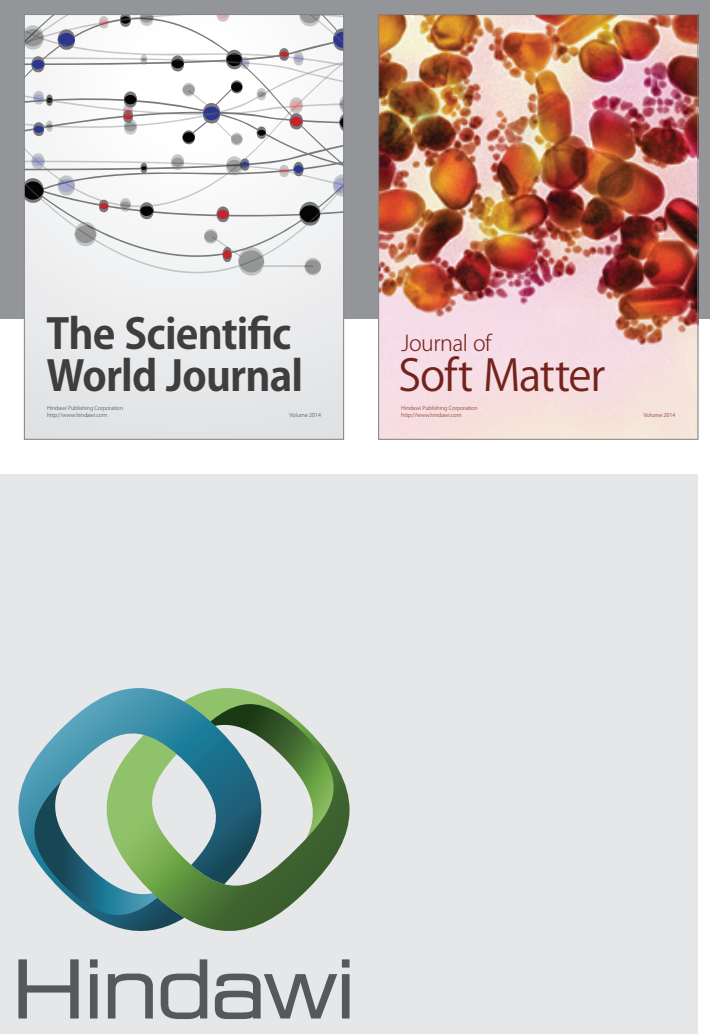

Submit your manuscripts at

http://www.hindawi.com
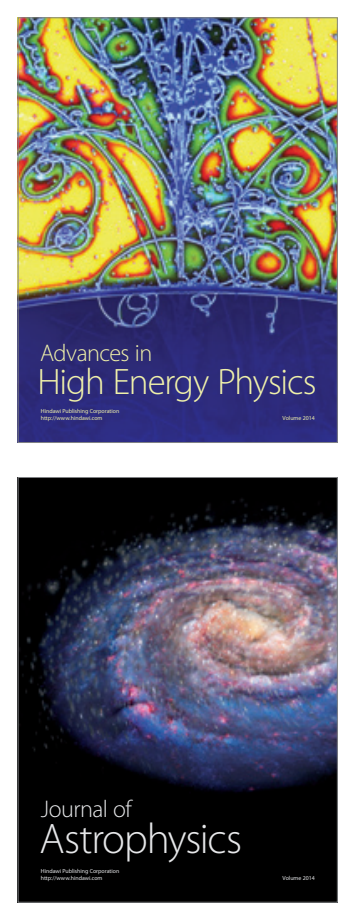
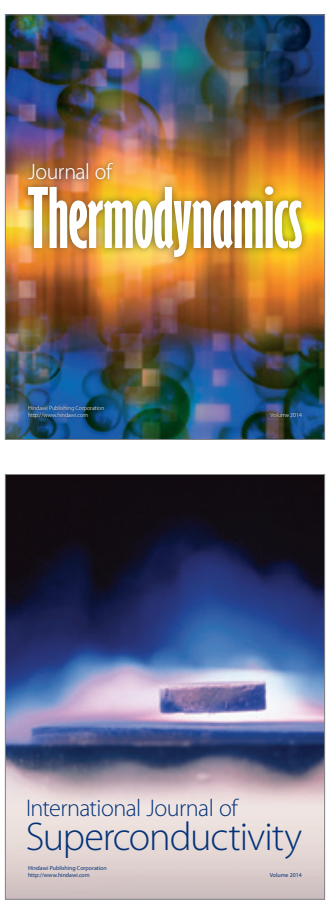
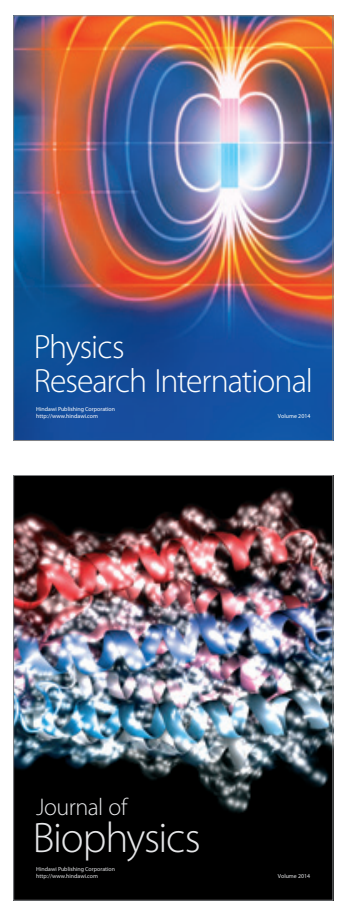
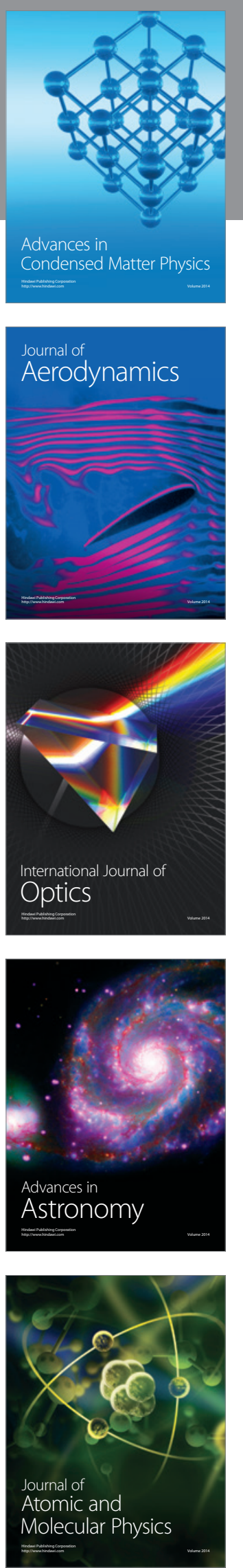